\title{
EDITORIAL
}

\section{The New Heart Failure Diet: Less Salt Restriction, More Micronutrients}

\author{
Michael B. Rothberg, MD, MPH', and Senthil K. Sivalingam, $M D^{1,2}$ \\ 'Division of General Medicine and Geriatrics, Department of Medicine, Baystate Medical Center, Springfield, MA, USA; ${ }^{2}$ Tufts University School \\ of Medicine, Boston, MA, USA.
}

$\mathrm{J}$ Gen Intern Med 25(10):1136-7

DOI: $10.1007 / \mathrm{s} 11606-010-1254-8$

(c) Society of General Internal Medicine 2010

$\mathrm{T}$ he Western diet has been implicated in a number of chronic diseases, including heart disease, diabetes and cancer. Patients have eagerly sought medical guidance on the proper diet, and although certain food elements appear to increase the risk of certain diseases, offering up specific dietary advice can be a treacherous endeavor. For one thing, diet is notoriously difficult to study. The effects of diet may take years to develop, few volunteers are willing to engage in randomized trials of diet for long periods of time, and protective factors identified in cohort studies rarely stand up to randomized trials. Not surprisingly, diet advice predicated on observational studies or pathophysiological mechanisms rather than randomized trial evidence may turn out to be ineffective (e.g. avoiding eggs) ${ }^{1}$ or even harmful (e.g. replacing the saturated fat in butter with margarine rich in trans-fats). ${ }^{2}$

Admitting that our past advice was wrong is uncomfortable and may also make patients less inclined to trust medical recommendations in the future. This may explain why the American Heart Association (AHA) was slow to rescind the recommendation to limit dietary fat due to its effects on total blood cholesterol levels. This recommendation has stood at the heart of public policy for decades ${ }^{3}$ but now appears to have been misguided. ${ }^{4}$ In fact, the vilification of fat may have encouraged increased consumption of sugar, which the AHA now recognizes as a possible cause of obesity, diabetes and heart disease. ${ }^{5}$

The notion that the lives of 6 million Americans living with heart failure might be improved by prudent dietary choices is appealing. Despite advancements in pharmacological therapy, heart failure remains the most common cause for both hospital admission and readmission among Medicare patients, with one quarter of heart failure patients re-admitted within 30 days after hospital discharge ${ }^{6}$ Dietary indiscretion (i.e. high salt intake) is often cited as a cause of heart failure exacerbation and hospital admission. ${ }^{7}$ Consequently, the AHA recommends that heart failure patients maintain a low sodium diet, but acknowledges that the recommendation is based on expert consensus only. ${ }^{8}$

In this issue of JGIM, Lemon et al. ${ }^{9}$ report that in a national sample of heart failure patients, the public is beginning to internalize the AHA message. Average daily sodium intake was

Published online February 12, 2010
$2.7 \mathrm{gm}$ for those with heart failure versus $3.0 \mathrm{gm}$ for the general population; but only $1 / 3$ of heart failure patients consumed less than $2 \mathrm{gm}$ of sodium. The authors highlight the difficulty in complying with a low salt diet and call for large-scale efforts to reduce dietary sodium. Should "low-salt" now replace "low-fat" and "low-cholesterol" on package labeling? Not so fast.

Heart failure is characterized by decreased cardiac output and resultant activation of the renin-angiotensin-aldosterone system. High circulating levels of aldosterone stimulate the kidney to retain salt and water. In such a state, salt restriction appears to make physiological sense, with the understanding that water follows salt, causing interstitial edema. Experimental evidence, however, suggests that at least for subjects who are not sodium deprived, high sodium intake expands intravascular volume via fluid shift from the interstitial space without increasing total body water. ${ }^{10}$ Conversely, in the setting of a medication regimen that includes agents that block the renin-angiotensin-aldosterone system as well as high-dose loop diuretics, there is little direct evidence that sodium restriction can improve heart failure symptoms. In fact, increasing doses of diuretics can compromise renal perfusion, further stimulating the renin-angiotensin-aldosterone system and worsening edema. Simultaneous activation of vasopressin causes retention of free water, leading to hyponatremia.

Amidst this altered hormonal status, is it possible that a normal sodium diet, combined with appropriate reninangiotensin-aldosterone system blockade, high dose diuretics and fluid restriction could actually restore intravascular volume and renal blood flow, paradoxically facilitating renal sodium and water excretion? In fact, two well-done randomized trials now suggest that this may be the case. Paterna et al., studied 232 patients with compensated heart failure following hospitalization. ${ }^{11}$ Patients were randomized to receive either a low (1.8 gm) or "less restricted" sodium (2.8 gm) diet plus high-dose oral furosemide (250-500 mg bid) combined with a $1000 \mathrm{ml}$ fluid intake for 180 days. At the end of the study, the normal sodium group had fewer readmissions ( $8 \%$ vs. $26 \%, \mathrm{p}<0.05$ ), as well as lower levels of brain natriuretic peptide, aldsoterone and plasma renin activity. A second study by the same group, featuring 410 patients recently hospitalized for heart failure, randomized patients to different levels of sodium intake (1.8 vs. $2.8 \mathrm{gm}$ ), fluid intake (1000 cc. vs. $2000 \mathrm{cc}$.) and furosemide dose (125 mg vs. $250 \mathrm{mg}$ bid) to assess the impact of each. ${ }^{12}$ Both higher sodium intake and lower fluid intake, and to a lesser extent high dose furosemide, were strongly associated with decreased readmissions and improved levels of brain natriuretic peptide, aldosterone and plasma renin activity at 
180 days. Such high doses of furosemide are not commonly used in practice and might be considered ototoxic or nephrotoxic. Despite this, renal function was preserved, and those consuming $2.8 \mathrm{gm}$ of sodium actually had improvement in creatinine clearance compared to patients on the low sodium diet. Before these studies can be incorporated into practice, they should be repeated by other investigators, perhaps including lower doses of diuretic or higher intake of sodium, but they should not be ignored.

Just as the wisdom of the 2 gram sodium restriction is coming under question, the role of other micronutrients in heart failure is beginning to be appreciated. The study by Lemon et al. also found that the vast majority of patients with heart failure follow a diet deficient in calcium, magnesium and potassium. ${ }^{9}$ Heart failure is now recognized as a systemic illness in which neurohormonal activation mediates release of pro-inflammatory cytokines, causes oxidative stress in a variety of tissues and induces a catabolic state with loss of soft tissue and bone known as cardiac cachexia. ${ }^{13}$ In this model, micronutrients such as calcium, magnesium, selenium, zinc, and vitamin D may play a more important role than previously thought. Elevated levels of aldosterone and chronic use of loop diuretics increase urinary excretion of calcium and magnesium, leading to secondary hyperparathyroidism, which in turn depletes calcium from bone and drives calcium into cells, increasing oxidative stress. The whole process may be exacerbated by vitamin D deficiency, which now appears ubiquitous in northern latitudes. Other micronutrients, including selenium, zinc, thiamine and coenzyme $\mathrm{Q}$ also appear to be reduced in heart failure patients.

How much of each is required, and will diets rich in micronutrients serve to improve heart failure outcomes? A recent small study examined the effect of a combination of micronutrient supplementation on left ventricular function and quality of life in elderly patients with stable systolic dysfunction $(\mathrm{EF}<35 \%)$ due to ischemic heart disease. ${ }^{14}$ Thirty patients were randomized to receive a combination of calcium, magnesium, zinc, copper, selenium, vitamins A, C, D, E, B6 and $\mathrm{B} 12$, thiamine, riboflavin, folate, and Coenzyme $\mathrm{Q}$ or placebo for 9 months. At the end of the study, the treated group had reduced LV volumes, increased LVEF (+5\% p<0.05) and improved quality of life scores; placebo recipients exhibited no changes. The sample size was small and included only elderly patients with heart failure due to ischemic heart disease. The intervention contained 10 different micronutrients at doses ranging from $1 / 3$ to 200 times the recommended daily intake. Given these limitations, the study offers little guidance as to which of these nutrients is essential or in what doses they should be given, but it does suggest the need for further investigation in this area.

There is no doubt that lifestyle elements, including diet and exercise, can have profound effects on the chronic diseases plaguing our modern society. Randomized trials have demonstrated the dramatic impact of combinations of diet and exercise on cardiovascular disease and diabetes. To extend these recommendations to heart failure, we need more randomized trials that investigate the effects of modulating various components of diet, fluid restriction and nutritional supplements. Given the high rates of readmission and mortal- ity in heart failure, important clinical endpoints can be achieved in months, studying hundreds of patients, in contrast to the years and the thousands of patients required to demonstrate the benefits of an additional treatment for coronary artery disease. ${ }^{15}$ Considering the persistently high morbidity and costs associated with heart failure, it is time to abandon our long-held beliefs about the heart failure diet and begin gathering evidence.

Corresponding Author: Michael B. Rothberg, MD, MPH; Division of General Medicine and Geriatrics, Department of Medicine, Baystate Medical Center, 759 Chestnut Street, Springfield, MA 01199, USA (e-mail: Michael.RothbergMD@baystatehealth.org).

\section{REFERENCES}

1. Hu FB, Stampfer MJ, Rimm EB, et al. A prospective study of egg consumption and risk of cardiovascular disease in men and women. JAMA. 1999;281:1387-1394.

2. Mozaffarian D, Katan MB, Ascherio A, Stampfer MJ, willett WC. Trans fatty acids and cardiovascular disease. N Engl J Med. 2006;354:1601-1613.

3. Gotto AM Jr, Bierman EL, Connor WE, et al. Recommendations for treatment of hyperlipidemia in adults. A joint statement of the Nutrition Committee and the Council on Arteriocslerosis. Circulation. 1984;69:1065A-1090A.

4. Mente A, de Koning L, Shannon HS, Anand SS. A systematic review of the evidence supporting a causal link between dietary factors and coronary heart disease. Arch Intern Med. 2009;169:659-69.

5. Johnson RK, Appel LJ, Brands M, et al. Dietary sugars intake and cardiovascular health: a scientific statement from the American Heart Association. Circulation. 2009; 120:1011-1020.

6. Jencks SF, Williams MV, Coleman EA. Rehospitalizations among patients in the Medicare fee-for-service program. $N$ Engl $\mathrm{J}$ Med. 2009;360: 1418-28.

7. Tsuyuki RT, McKelvie RS, Arnold JM, et al. Acute precipitants of congestive heart failure exacerbations. Arch Intern Med. 2001;161:2337-42.

8. Hunt SA. ACC/AHA 2005 guideline update for the diagnosis and management of chronic heart failure in the adult: a report of the American College of Cardiology/American Heart Association Task Force on Practice Guidelines (Writing Committee to Update the 2001 Guidelines for the Evaluation and Management of Heart Failure). J Am Coll Cardiol. 2005;46:e1-82.

9. Lemon S, Olendzki B, Magner $\mathbf{R}$ et al. The dietary quality of persons with heart failure in NHANES 1999-2006. J Gen Int Med. 2010. doi: 10.1007/s11606-010-1296-y.

10. Heer M, Baisch F, Kropp J, Gerzer R, Drummer C. High dietary sodium chloride consumption may not induce body fluid retention in humans. Am J Physiol Renal Physiol. 2000;278:F585-95.

11. Paterna S, Gaspare P, Fasullo S, Sarullo FM, Di Pasquale P. Normalsodium diet compared with low-sodium diet in compensated congestive heart failure: is sodium an old enemy or a new friend? Clin Sci (Lond). 2008; 114:221-30.

12. Paterna S, Parrinello G, Cannizzaro S, et al. Medium term effects of different dosage of diuretic, sodium, and fluid administration on neurohormonal and clinical outcome in patients with recently compensated heart failure. Am J Cardiol. 2009;103:93-102. Epub 2008 Oct 17.

13. Alsafwah S, Laguardia SP, Arroyo M, et al. Congestive heart failure is a systemic illness: a role for minerals and micronutrients. Clin Med Res. 2007;5:238-43.

14. Witte KK, Nikitin NP, Parker AC, et al. The effect of micronutrient supplementation on quality-of-life and left ventricular function in elderly patients with chronic heart failure. Eur Heart J. 2005;26:2238-44. Epub 2005 Aug 4.

15. Kent DM, Trikalinos TA. Therapeutic innovations, diminishing returns, and control rate preservation. JAMA. 2009;302:2254-6. 